Historic, Archive Document

Do not assume content reflects current scientific knowledge, policies, or practices. 



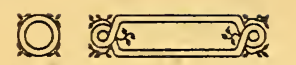

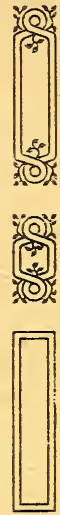

\section{ESTABLISHED 1906}

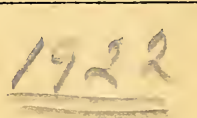

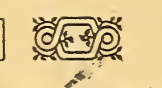

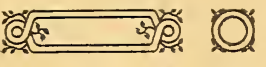
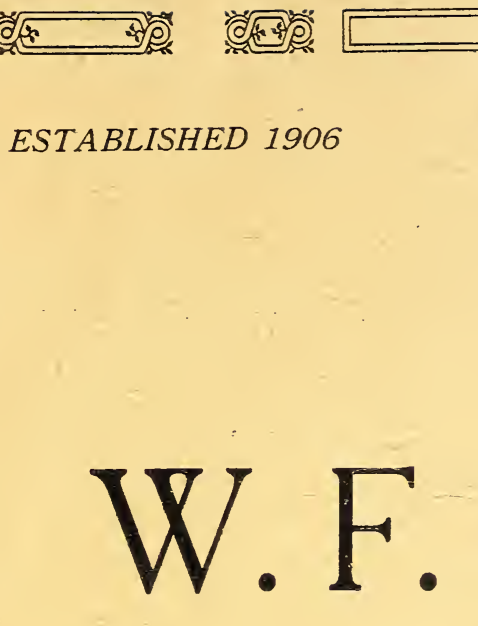

Hardy Phlox Book
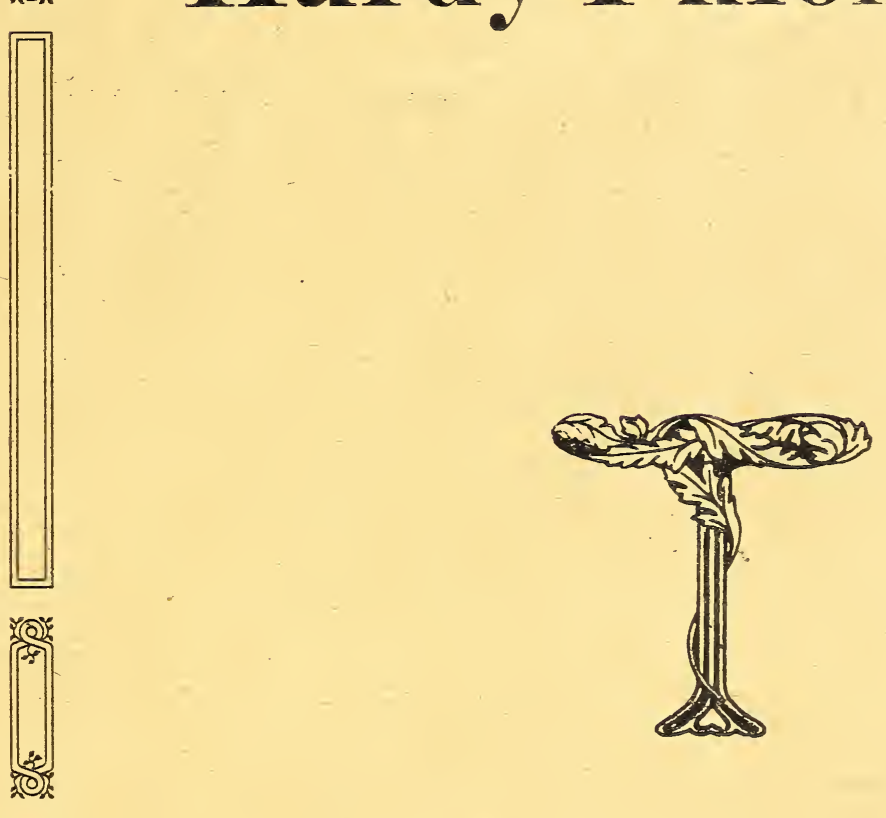

.
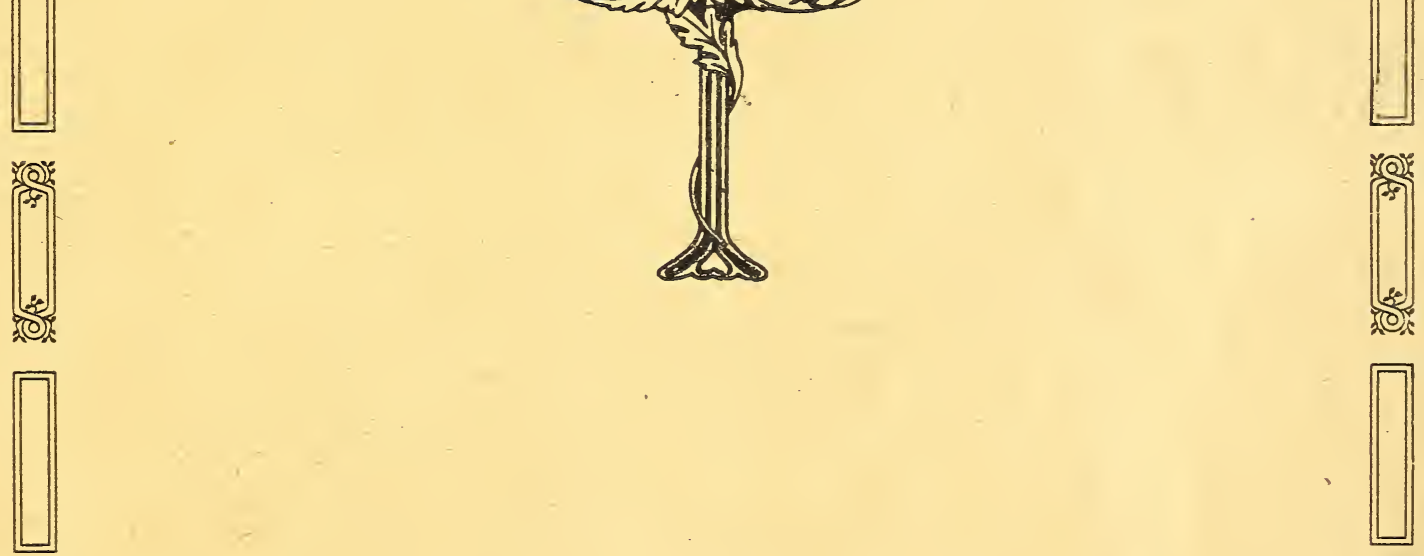

(ृ)

Address: State Hospital Binghamton, N Y. 


\section{BUSINESS TERMS}

We are selling as cheap as the cheapest and the stock is as good as the best, hence feel assured you will be pleased.

Our terms are net cash as the margin is so small that we can not carry a book account. Send remittance with order.

Please write your name and address plainly, look over your order after finishing'in order to be sure that everything is on the list, also your name and address. It is surprising how many people forget to write the address on the letter.

We charge nothing for packing and delivering to station but if plants are shipped at wholesale prices extra charges for packing will be charged.

Should there be any error in the order, advise me at once and it will be corrected.

We will not substitute, unless requested. If we have not what you want, you will receive your money back.

Three plants of one variety will be sent at dozen rate but less amount must be paid for at single rate.

I guarantee to send out strong and healthy plants and that customers will receive fresh and live plants and true to name. Beyond that I can not guarantee. Should they not be true to name, I will replace them but can not hold myself responsible if the plants do not grow after planting as I have no control over them after they leave me.

Small orders will be sent by Parcel Post, but anything over 1 dozen should be sent by express.

If plants can reach their destination in 24 hours, an extra charge of $15 \mathrm{c}$ should be added per dozen; if longer on the way, the charge will be more according to distance if shipped by Parcel Post.

I do not grow any plants in pots, hence better plants will be shipped.

Tell me how you want your plants shipped. If, however, no statement is made, I will send to the best advantage.

I send out no catalogue except on request. 


\section{MODERN HARDY PHLOX}

Are still the leaders for cemetery work, rock gardens, old fashioned gardens, up-todate shrubbery and border. The demand keeps pace with the improvement of them in all landscape work-large and small. That it gave me a great deal of encouragement to gather together under one cover all the good things from home and abroad.

I have spared neither time nor patience thoroughly selecting and crossing to get only the best and in looking the list over you will readily perceive what the success has been, so that at the present there is no firm who can show a larger list of this flower.

What was said in my former list bears repeating, that all Phlox are on trial at least two years and if not entirely satisfactory, longer, then, if found wanting, thrown out and only the best retained.

Before going further I would like to call attention to this fact, that all my Phlox, as they stand in the garden, have been grown under my own supervision for at least two years on the spot itself. The soil is a sandy loam, the plants have never had any artificial watering, and they have been left outside in the ground throughout the winter without any cover but a very thin layer of horse manure. The garden itself is fully exposed to the inclemency of the weather, against which it has had no protection whatever. I have by this way of growing plants eliminated every plant not a complete hardy variety.

Before planting Phlox the soil should be well enriched with manure as they usually remain on the ground for several years and are strong growers.

When transplanting or when Phlox are received and the soil is prepared make the hole large enough so the roots can be spread out and the crown of the plant planted two inches below the surface of the soil. Pull the soil over the plant and press down hard using your hand or foot to press the soil down hard, for a great deal depends on this part of the operation for the life of the plant, then give them a light mulch of manure or leaves to cover them through the winter. In the spring the mulch may be left between the plants but should be removed from the crown of the plants.

The transplanting of this stock is an easy matter, and the Phlox are bound to grow and give satisfaction wherever planted almost without any care or labor at all. guarantee good, strong stock from the field. Should a plant when received prove unsatisfactory for one reason or another, it can be returned at my expense if care is taken that the plant is not spoiled by the drying of the roots or in any other way.

I aim to only grow the absolute best stock and as I always will have the supervision of the filling of orders and shipping myself, the patrons call rest assured that they will get both good stock and the stock they ask for.

The planting of Phlox should be done from the latter end of September until a very 
hard frost stops all plant-life. But the order for. Phlox should be given as early as possible. Fall is the proper time to transplant Phlox, but the work can be done early in spring. The plants will be strong and all of such a size that they will give absolute satisfaction the first year as to growth and flowers. No roots will be sent out without first having been tested and flowered. It is the aim to avoid all disappointment on the part of the buyer.

As will be seen, it is my intention under the preparations to put my business on such a base of solidity and honesty as to the good quality of the stock that I trust everybody who makes a trial will find that they never have been served as good before and that they are fully and amply repaid for their expense.

When the soil gets dry in the summer water should be applied to the roots. Not sprinkled, but let the water run on the ground and if thoroughly wet left until dry again.

Mildew may attack the plants in the summer. Apply powdered sulphur dusted on the foliage in the morning when dew is on the foliage will arrest the disease.

Red Spider is another pest that sometimes makes its appearance but if the plants are watered regularly, spider will not do any harm. Spiders are controlled by syringing the plants with water reaching well under the foliage.

Fourteen inches is a good distance to plant Phlox, and if the clumps get too large they should be devited and transplanted, the soil being prepared the same as newly set plants.

There is a lot of choice varieties added to my former list. I made an effort to describe as near as the color appeared to my eye, but as the list is such a proportionate one I did not attempt to name them all but added them by number, leaving them to be named by you if you so desire.

\section{W. F. SCHMEISKE,}




\section{Paniculata (Decussata)}

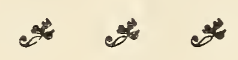

In Flower

ALHAMBRA. Pink; white halo; very effective.

July 30 ATHIS. Deep pink, overlaid salmon. Crimson eye................. July 29 ALBATRA. Pure white. Good color. $11 / 2$ feet..................... 5 ARGON. Light lavender. Shaded white. $11 / 2$ feet................. July 28

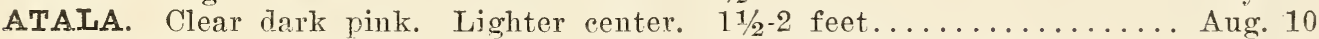
ASTIR REHU. Violet purple, half of each. Petal lighter. 3 feet....... July 26 AMPHITRYON. Deep lilac, suffused with white. Deep eve. 21/2 feet.... July 26 ANTHON MERCIE. Grayish lavender, pale pink eye. A gorgeous flower.

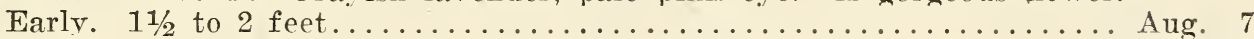
ASPASIF. Lilac, shaded white; white center. 3 feet................ Aug. 10 ALBION. White, a faint pink center. Dwarf. $2 \frac{1}{2}$ feet.................... July 7 AQUILON. Beautiful carmine rose. crimson eye................... July 20 ARNOID TURNER. Fiery crimson scarlet. $2 \frac{1}{2}$ feet............. July 15 AGIAE ADAMSON. The same as 152 (Michael Cervantea).............. Aug. 5 ANDREAS HOFFER. An early flowering pure white.................. July 20 A. MICHAUX. Flowers large, very fine; deep, tender rose with carmine eve.

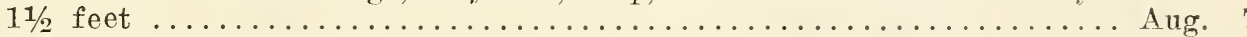
AURORA BOREAIIS. Salmon pink, white halo, dark center, petals striped, lively colored. $11 / 2$ feet................................... 28 BROGNIART. Very light lavender pink, white edges, deep eye. $11 / 2$ feet. Aug. 5 BERANGER. White suffused pink, bright crimson eye.............. Ang. 7 BICOLOR. Pink. Carmine eye. $21 / 2$ feet.......................... 1 BOULE DE FEU. Salmon scarlet. Dark crimson eye. 2 feet........... July 15 BOUQUET FLEURI. White overlaid pink. Analine red eye.......... July 30 BOUQUET DE FIEURS. White, overlaid pink, lilac center, small flower.

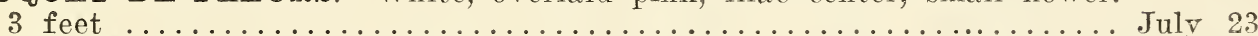
BOUNTAIN. Deep rose. Deeper center, $21 / 2$ feet............... July 26 BRIDESIMAID. Pale rose pink, light crimson center. Beautiful. 3 feet... July 20 BRILIIANCE. Brilliant scarlet. Slightly penciled lighter. 3 feet...... July 28 BRUNETT. Fiery crimson. Very effective color. Brilliant and showy. 21/2

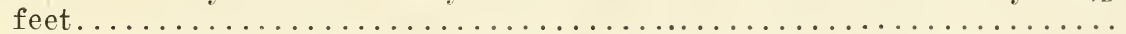
CONSUT T TROST Brilliant crimson Brilliant color 11/2 feet ....... CAMTRON Salmon nink chan CAPT. WILHELIMY. White, tapering pink toward center; crimson eye. $21 / 2$ feet. Midseason. CARON d'ACHE. Bright carmine rose. Deep center, large flower. $2 \frac{1}{2}$ feet. July 22 CHAIMPS d'ELISSEES. Bright rose magenta on effective shade........ July 26 CHARIES DARWIN. Handsome deep pink. Purple eye, lighter lake.... Aug. 10 COLIBRI. Grayish lavender, crimson eye, tapering white toward the edges. Aug. 5 COMMONWEALTH (on order of 24) but striped. Good flowers. Late, fine.

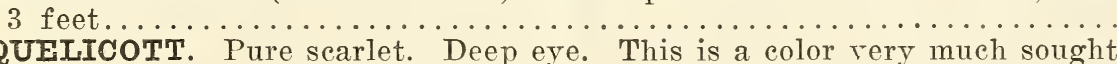
after by many. $21 / 2$ to 3 feet................................... 20 CREPUSCÚLE. Lilac, shaded lighter toward center. Large flower. 2 feet. July 22 CROSS OF HONOUR. Light lavender. Lighter stripes. Very odd. 3 feet July 25 CYCION. White suffused pink, lilac crimson center. Large flower....... Aug. 1 CZARINA. Fine white, very late. Large clusters. 3 feet............. Aug. 15 DE MIRIBEL. Deep lilac purple. Light halo. Large flower. $11 / 2$ feet.... Aug. 1 DUPAUloup. Creamy white ............................... 7 DAWN. Soft pink, deep pink center. $1 \frac{1}{2}$ feet. Very pretty shade....... July 15 DAILY. Light rose. Deep center. Deeper eye. Fine color. 11/2 feet.... July 22 DELIVERANCE. Deep lilac with Tyrion red eye. Very effective........ Aug. 1 DERWISH. Rose lilac, mottle white. 2 feet................... July 25 
DR. S. ANDRAL KILMER. Variegated red on white ground, some flowers heavy, some lighter, variegated, $1 \frac{1 / 2}{2}$ feet; flower $11 / 2$ inches.......... Aug. 1

192 DR. CHARLES G. WAGNER. Pinkish white, with light purple center run-

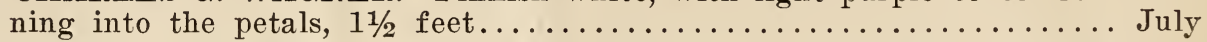

327 ELIZABETH CAMPBELL. Very bright salmon-pink, with lighter shadings

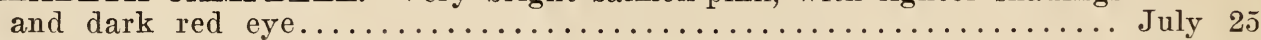

EMBRASEMENT. Copper salmon center erimson. Aug. 1 ESCLARMONDE. Rosy lilac; aureole of white; purple violet center....... Aug. EXQUISITE. Deep lilac; light halo; large flower. 2 feet.....................
ECLAIREUR. Reddish carmine, dark eye. Lighter halo. Very showy.

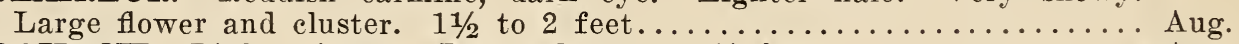

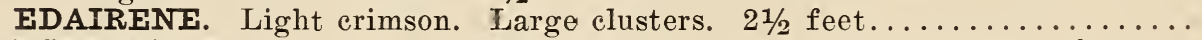
ESPERANCE. Lilac rose. Deep lilac center. Immense clusters. 2 feet....
ETNA. Bright crimson, deep crimson eye. Some flowers changing to lighter

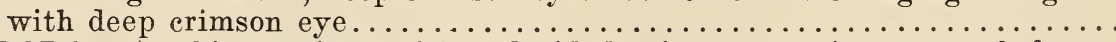
EUROPA. A white variety with a decided crimson-carmirne eye. 3 feet. FRAU DORA UMGELLER. Tall. Very rich, deep rose; a strong free-flow-

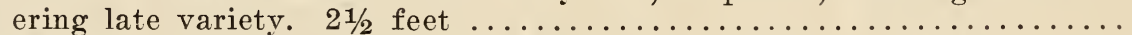
FANTOME. Heliotrope. Deep lilac center. Large flower. $2 \frac{1}{2}$ feet......

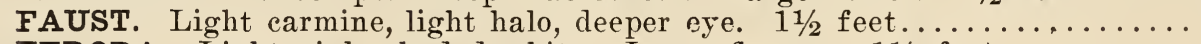
FEDORA. Light pink, shaded white. Large flowers. $11 / 2$ feet.......... FRAU ANT. BUCHNER. Pure white, very large flowers, strong grower....

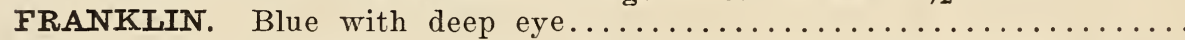
GRAFF VON UNGERER. White overlaid pink with deep crimson center.. Aug. flower $21 / 2$ inches................................................. GEN. CHANZY. Brilliant cherry. Deep eye. One of the best in its color.

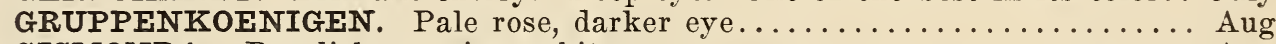
Aug. 1

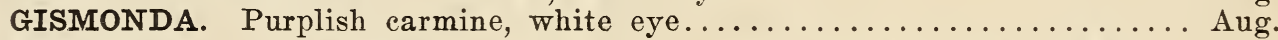
HAMBEAN. Orange crimson. Maroon eye. Good cluster. Late. $21 / 2$ feet. HANNY PFLEIDERER. Pale rose tinted salmon, beautiful carmine eye... HENRY MUERGER. White with pink eye. Large flower. 3 feet..

Aug.

Aug.

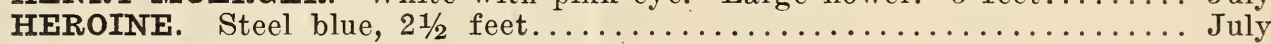
H. O. WIGERS. White with analine red eye.

July 30 HUXIEY. Light carmine rose. Deeper center. 3 feet.................. 7 INSPECTOR ELPEL. Tender Rose, with bright crimson carmine eye..... INDEPENDENCE. White, free bloomer. Good flower. $2 \frac{1}{2}$ feet........

JAMES BENNETT. Light pink, tinted salmon. Large crimson center. 3

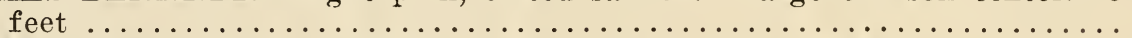
JAMES GALLOWAY. Brilliant cherry. Dark eye. $2 \frac{1}{2}$ feet.......... July 25 JOCELYN. The same as Coccinea. Bright salmon red. Very brilliant.

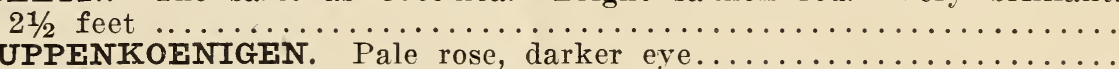

\section{KUCKEN. Salmon, pink, dark rose center, large flowers}

July 20 Aug. 5 LAMARTINE. Tall; very bright maganta, with a large white center. Very

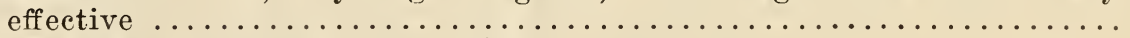

LA CYGNE. Pure white. Fine spikes. 1 foot..................... July LE FEU DU MONDE. Bright salmon red.................... Aug. 7 LAMBOIRE. Brilliant orange salmon, crimson center. $2 \frac{1}{2}$ feet. Fine... July 27 IA POLE DU NORD. White, crimson eye., $21 / 2$ feet.................. July 25 IA REVE. White, pink penciled. 3 feet. . 
IE MAHDI. Deep purple. Changes to deep blue with age and penciled.

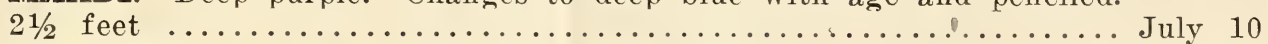

LE SESOSTUE. Deep rose. Crimson center. 2 feet............................ 20

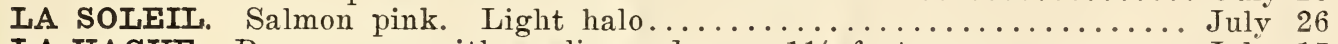

I.A VAGUE. Pure mauve with analine red eye. $11 / 2$ feet............... July 15

LUMIINEUX. Crimson orange. Lighter lake. Bright carmine eye. 3 feet.

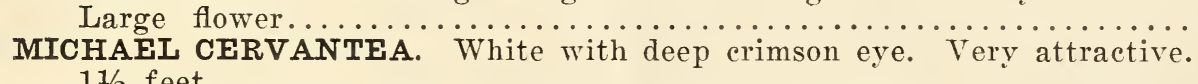

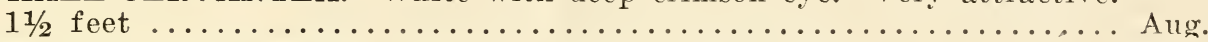
MME. PAPE CARPENTER. Brilliant cherry. Small flower............ Aug. MONTCERVIN. On order of Coquelicot. Not as brilliant. $1 \frac{1}{2}$ feet...... Aug. MARIE KUPPENHEIM. White. Good flower. $1 \frac{1}{2}$ feet............ July 15

MARIETTA. Purple. Large flower. Light halo....................... 22 MARS LA TOUR. Pale pink. Deep rose eye. $21 / 2$ feet............. July 30

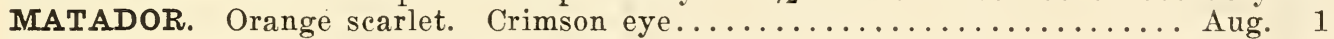
MITEOR. Salmon rose, pale pink lake. 2 feet....................... 30 MINERVA. Deep lavender pink. Tyrion red eye. 2 feet............ Aug. 1 MOLIERE. Salmon rose. Deep rose eye................... July 26 MONTAGNARD. Pure crimson. Very bright. $21 / 2$ feet............... July 20 WANADIS. Light violet, mottled white with a bright purple eye........

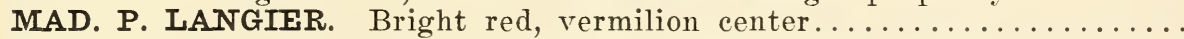
MRS. DWYER. Pure white. Analine red center. Large flower. 3 feet... July 31 MRS. JENKINS. Good creamy white. $1 \frac{1}{2}$ feet................... 5 M. WISE. Deep purple. $11 / 2$ feet............................. 26 MRS. W. F. SCHMEISKE. Light rose, white center, 11/2 feet, flower 11/2 inches

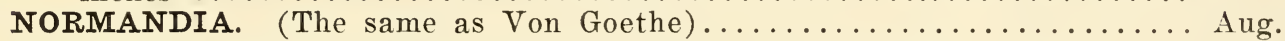
NORWOOD. Bright Magenta crimson. Dark eye. $2 \frac{1 / 2}{2}$ feet........... Aug. OBERGARTNER WITTIG. Bright magenta, with erimson carmine eye;

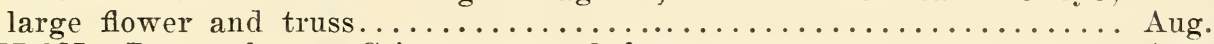
OBERON. Deep salmon. Crimson eye. 3 feet....................... OFFENBACH. Deep pink. Lively color. 2 feet................. Aug. PHILIBERT AUDENBRAND. Tall. Pure white with carmine center.

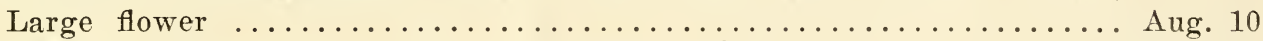
PANTHEON. Deep salmon. Pale rose center. 3 feet...............July 22 PEACH BLOW. Light pink. White lake. $11 / 2$ feet.................. July 1 PINK BEAUTY. Delicate pink, large trusses lasting a long time. Very

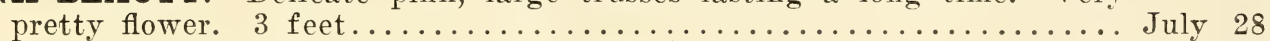
PLUTO. Deep rose; light center. Good truss. 2 feet............... July 22 PROF. SCHIIEMANN. Pure mauve, with crimson carmine eye. $1 \frac{1}{2}$ feet. July 15 QUILQUOI. Lavender. Purple eye. Large flowers. $1 \frac{1}{2}$ feet......... July 15 RAYOUMAUT. Variegated, very odd, crimson purple with lavender stripes.

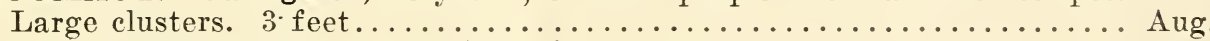
RICHARD WALLACE. Tall white; violet center. 3 feet............. Aug. RICHEPIN. Slightly suffused rose.. Crimson scarlet eve............. A ug. ROBERT WARNER. Rose; deeper center. Large truss and flowers. 3 feet. Aug. 1 ROSE CHARIMONT. Light violet. Deep purple eye. $21 / 2$ feet......... July 22 ROSE LILAC. Small flowers. Large clusters.......................... 26

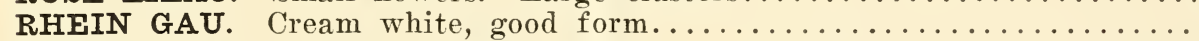

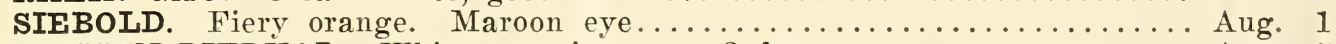
SAISSON IIERVAL. White, carmine eve 2 feet.................. 5 SIMPLON. Deep salmon. Crimson eye. $2 \frac{1}{2}$ feet............... July 27 SUFFRAGE. Light crimson, deep eye............................ 10 SUNSHINE. Deep pink, penciled white. Rose center. $21 / 2$ feet....... July 27 SYLPHIDE. White, good truss and flowers. 2 feet...................... 25

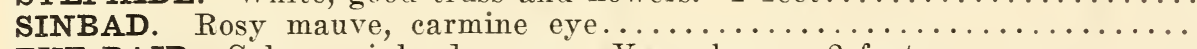

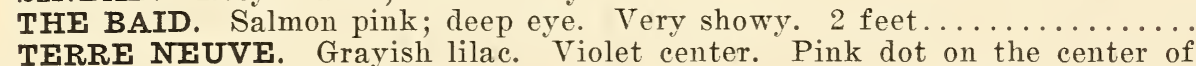

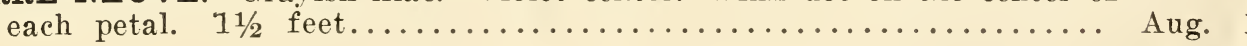


In Flower

81 VENGEUS. Deep crimson. Maroon ere. 2 feet................... Aug. 5

58 VENUs. Deep rose. Tyrion eye. Late. $1 \frac{1}{2}$ feet......................... 15

117 VON HOCHBERG. Brilliant maroon. Very dark. Some petals spotted

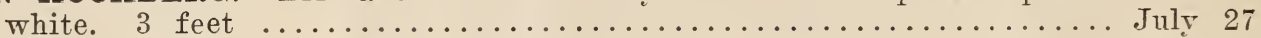

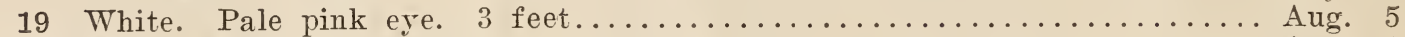

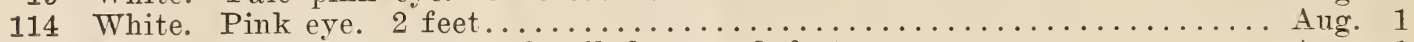

141 White, very faint stripes. Small flower. 3 feet.................. Aug. 1

35 WM. MEURET. Brilliant cherry. Crimson eve. 3 feet.............. Aug. 1

64 WM. RAMSEY. Light rose. Deep eye. Lighter lake. Large flower....... July 22

92 W. VON GOETHE.. Tyrion rose, suffused carmine; carmine eye......... July 22

140 DANIEL LESSEUR. Blenish lavender. White lake. Large flower. 21/2 ft. July 28 MY SELECTION OF THE LIST NOT LABELED, \$1.00 PER DOZ. SPECIAL PRICES ON LARGER LOTS.

$20 \mathrm{c}$ each.

$\$ 2.00$ per dozen

\section{LATEST INTRODUCTIONS}

W. C. EGAN. One of the finest Phloxes yet introduced, and while the individual flowers, according to color chart, are of a delicate lilac, illuminated by a large bright solferino eye, the color effect as a whole is a pleasing shade of soft pink. In habit of growth it is strong and vigorous, producing immense panicles of flowers nine inches high by seven across and the individual flowers frequently measure $13 / 4$ inches in diameter.

MRS. W. E. FRYER. White, a faint pink center, strong growth, good truss.

MANZELBRUNNER. One of the white center sorts.

172 White overlaid with pink, lilas center, 1 foot, flower $1 \frac{1}{2}$ inches.

176 White, faint pink marking in center of flower, 14 inches.

179 Deep reddish purple with light halo, 20 inches.

180 Grayish white, light pink tube, $2 \frac{1}{2}$ feet.

181 White, faint pink tube, large flower, good truss, 20 inches.

182 Pink, running toward purple, crimson eye, 14 inches, flower $1 \frac{1}{2}$ inches.

183 Deep rose, light crimson eye.

186 Crimson scarlet, crimson eye, $2 \frac{1}{2}$ feet.

187 Crimson purple.

188 Bright, pink, light crimson eye, 16 inches.

189 Clear white, faint trace of pink in center in early stage, $1 \frac{1 / 2}{2}$ feet.

190 White, faint pink center, flower 1 inch.

191 Lavender pink, crimson scarlet eye. $1 \frac{1}{2}$ feet, flower $1 \frac{1}{2}$ inches.

193 Lilac, deeper center, 2 feet.

194 Brilliant crimson, 11/2 feet.

195 White mixed with gray, giving gray appearance, light crimson eye.

196 Light purple throughout, large flower, 14 inches.

197 Bluish lavender, lilac center, 2 feet, flower $1 \frac{1}{2}$ inches.

198 Pink lavender, crimson eye, 11/2 feet, flower $11 / 2$ inches.

199 Lavender, changing to deep lavender toward center, flower 11/2 inches, plant 11/2 feet.

200 Lively light purple, light halo with deep eye, $1 \frac{1 / 2}{2}$ feet.

201 Lavender. Open star-like flower.

203 Grayish lavender, deeper toward center, crimson eye, $11 / 2$ feet, flower 2 inches.

204 Improved La Reve, striped and penciled, pink on white, 1 foot, flower $11 / 2$ inches.

205 Deep crimson center, light pink petals, white edge, $11 / 2$ feet, flower $11 / 2$ inches.

206 Lively pink with traces of lilac, light halo, deeper eye, flower $11 / 2$ inches.

207 Lively crimson pink, light halo, deep crimson eye, heary flower.

208 Pink lavender, light halo.

209 Crimson scarlet, crimson eye.

210 Crimson, running lighter toward adge, white edge, $11 / 2$ feet, flower $11 / 2$ inches.

215 Very pale, lavender, deeper eye, 1 foot, flower, 1 inch.

217 Salmon pink, crimson center, light halo.

221 Deep lavender lilac. 
Deep magenta, light halo, deeper eye.

Tyrion rose, light halo, deep eye, grand cluster, 2 feet.

Deep lavender, lilac center, 2 feet, flower $1 \frac{1}{2}$ inches.

Lavender, light halo, lilac center, $1 \frac{1}{2}$ feet, flower $1 \frac{1}{2}$ inches.

Light lavender, light halo, lilac center.

Deep lavender, crimson eye.

\section{Light pink, rose eye.}

Deep lavender, crimson eye, 2 feet, flower $1 \frac{1}{2}$ inches.

Grayish white, crimson center, 2 feet, flower $1 \frac{1}{2}$ inches.

Light purple, 8 inches.

Lively lilac, light halo, 2 feet, flower $1 \frac{1}{2}$ inches.

Rose with white center, flower $1 \frac{1}{2}$ inches.

Light cerise, light halo, 2 feet, flower $1 \frac{1}{2}$ inches.

Carmine rose, crimson eye, slightly striped, lavender, 2 feet, flower $1 \frac{1}{2}$ inches.

Crimson purple, variegated lavender, 2 feet.

Deep rose pink, crimson eye, 2 feet, flower $1 \frac{1}{2}$ inches.

Crimson lilac, crimson eye, 2 feet.

Intense crimson maroon.

Pale lilac, light halo, light erimson eye.

Light crimson, deep crimson eye, $1 \frac{1}{2}$ feet, flower $1 \frac{1}{1} / 2$ inches.

Lively lavender, $1 \frac{1 / 2}{2}$ feet, flower $1 \frac{1}{2}$ inches.

Crimson pink, edge of petals white, $1 / 2$ feet, flower $11 / 2$ inches.

Lavender cerise, 2 feet.

Bluish lavender, light edge, white halo, $1 \frac{1}{2}$ feet.

Pale pink, crimson eye, 10 inch plant.

Rose pink, pink lilac eye, $1 \frac{1 / 2}{2}$ feet, flower $1 \frac{1}{4}$ inches.

Deep lavender, pink lilac eye, $1 \frac{1 / 2}{2}$ feet, flower $1 \frac{1}{4}$ inches. .

Tyrion rose, crimson eye, 2 feet.

Pale lavender, on order of "Hercine."

White, spotted and splashed crimson pink.

Crimson purple, light dot on each petal, 1 foot.

Crimson pink, crimson center, 2 feet.

Orange pink, crimson center, 1 foot.

Crimson, maroon center, $2 \frac{1}{2}$ feet.

Faint lavender, white center, $1 \frac{1}{2}$ feet, flower $1 \frac{1}{2}$ inches.

Bright cerise, white halo, cerise eye.

Pink lavender, crimson eye, 11/2 feet, flower 11/2 inches.

Pink, overlaid lavender, crimson eye:

Lavender, light halo.

White, crimson maroon eye, $1 \frac{1}{2}$ feet, flower $1 \frac{1}{2}$ inches.

Lavender, pnk and lilac, lilac eye, $11 / 2$ feet, flower $11 / 2$ inches.

Pale lavender, light halo, lilac eye, $1 \frac{1}{2}$ feet, flower $1 \frac{1}{2}$ inches.

Lilac, striped and penciled white.

Lilac cerise, white center.

Pale pink, white stripe on edge of each petal, 2 feet, flower 11/2 inches.

On order of 230 , but larger flower. Lilac pink, crimson eye. 11/2 feet flower $13 / 4$ inches.

Cerise pink, light halo, crimson eye, $1 \frac{1}{2}$ feet, flower $1 \frac{1 / 2}{2}$ inches.

White, splashed, penciled pink.

White, overlaid with lavender, flower $1 \frac{1}{2}$ inches.

Deep lilac, light halo, crimson purple eye, flower 11/2 inches.

Lavender, pink crimson eye, halo of white, flower $1 \frac{1}{2}$ inches.

Salmon rose, here and there crimson spot on edge of petal, crimson eye, flower $11 / 2$ inches.

Rose pink, white halo, flower 11/2 inches.

Rose lavender, white halo, flower $1 \frac{1}{2}$ inches.

Crimson purple, open flower, flower $1 \frac{1}{2}$ inches.

Grayish lavender rose, deep crimson, purple eye, flower 11/2 inches.

Pink, overlaid with gray, erimson eye, flower $1 \frac{1}{2}$ inches.

Lavender rose, rose stripes through center of each petal light érimson eye, flower $1 \frac{1}{2}$ nches. 
285 Deep pink, lavender erimson eye, 2 feet, flower $1 \frac{1}{2}$ inches.

288 Pink, overlaid lavender, light halo, erimson eye, large flower.

290 Lilac, light halo.

291 Varigated, lavender on white.

292 Large lilac, purple, crimson eye.

293 Calico, crimson purple.

294 Deep lavender, erimson, crimson eye.

295 White, penciled pink, very pretty.

296 Deep rose pink, white halo, bold, flower $1 \frac{1}{2}$ inches.

297 Large white with lavender eye, flower $1 \frac{1}{2}$ inches.

299 Crimson lilac, white halo, large flower.

301 Large white.

302 Deep crimson purple, erimson eye, slightly striped, large flower.

303 Purple, crimson eye, large flower.

304 Deep pink shading to lavender, light halo, light erimson eye, very large flower.

305 Crimson lilac, crimson eye.

306 White, faint cast of lavender.

\section{ABOVE 30c EACH, $\$ 3.00$ PER DOZ.}

It is impossible in some instances to find the correct description for the colors and in some instances the description is alike; however, the varieties are different. 


\section{EARLY PHLOXES}

100 BEAUTY OF MINDEN. White, ground flushed rose, deep center. Beautiful foliage overlapping one another in rows. Continuous bloomer. $2 \frac{1}{2}$

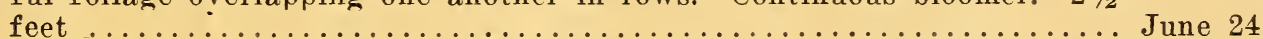

98 DR. HORNBY. White overlaid with pink. Crimson center. Good. $1 \frac{1 / 2}{2}$ to

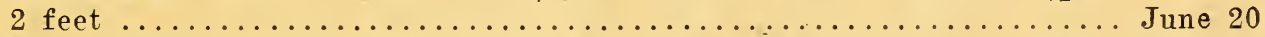

137 MISS LINGARD. White, delicate pink center. Beautiful flower. Good

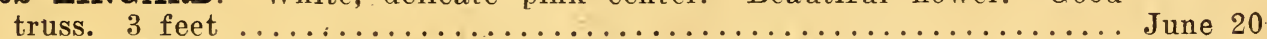

75 RINGLEADER. White, pink stripes. Deep center. $2 \frac{1}{2}$ feet.......... June 20

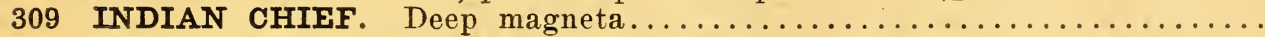

311 DIVARICATA CANADENSIS. An early flowering native. Species with lavender flowers. Blooming from early May until the Summer.........

These early phloxes ought to have a place in every collection. While they do not show the large clusters they more than make up the difference in quantity. The clusters are good, large heads, and flower the season through without letup. The foliage is shiny green, very showy and beautiful.

We can supply seeds of Phlox in the Fall which should be sowed as soon as received; it will come up the following Spring; from general collection at $\$ 1.00$ per ounce; from choice seedings, $\$ 2.00$ per ounce.

\section{A NEW RACE OF HARDY PHLOXES}

\section{(Phlox Arendsi.)}

At the great International Exhibition held in Londan, Mav, 1912. where this new type of Phlox received an Award of Merit no other new plant in the Hardy Perennial class attracted such great attention. It originated through the successful crossing of the earlyflowering popular Phlox Divaricata Canadensis with the showy hardy varieties of Phlox Decussata. The plants are of vigorous, branching habit, growing, according to the variety, from 12 to 24 inches high. Coming into flower during the latter part of May, they continue in good condition for nearly two months, producing a mass of flowers unknown in other types of Phlox.

AMANDA. The dwarfest of the collection, growing but 12 inches ligh, very bushy, flowers delicate lilac with deep centre.

CHARLOTTE. Grows 18 to 24 inches high, flower very large, pale lilac shaded pink with a prominent dark eye; one of the best.

KATHE. Stems loosely tranched, about 20 inches high; flowers purplish-rose with a dark eye surrounded by a lighter halo.

LOUISE. A strong-growing variety about 2 feet high; light lilac flowers with a carmine eye.

Price, 25 cts. each; $\$ 2.50$ per doz. One each of the 6 varieties for $\$ 1.25$. 
